\title{
ANALYSIS OF FLUID FLOW TRANSLATION CONVEYORS
}

\author{
Janis Viba, Vitalijs Beresnevich, Maris Eiduks, Lauris Stals, Kaspars Burbeckis \\ Riga Technical University, Latvia \\ janis.viba@rtu.lv, vitalijs.beresnevics@rtu.lv, maris.eiduks@rtu.lv, \\ lauris.stals@gmail.com, kaspars.burbeckis@gmail.com
}

\begin{abstract}
Fluid (water) flow translation motion conveyor (transporter) is being reviewed. In this device the actuator blades move in the plane axis parallel cycloidal motion, but the fluid flows away perpendicular to the rotation axis in translation direction. For this purpose the blade two component movement is synthesized by kinematics of the gear box: the first rotation takes place around the central axis of each blade, while the other rotation moves around this centre around the central axis of the transporter. The movement is designed in such a way that the central axis rotates twice as fast as the blades around its axles. The kinematics and dynamics of the transporter movement are analyzed, taking into account the characteristics of the drive motor and the blade interaction forces with fluid. The results of the analysis are shown in the graphs obtained by computer modelling. The possibility of creating a multi-element conveyor is being reviewed on the basis of one rotational element. In this case, it is possible to increase the efficiency of the system in such a way that the individual small conveyors in pairs operate in counter phase (rotates opposite). For transporter experimental investigations a special system is made inside the water tank. The system includes a rotating beam with a possibility to stick the devise in the end of this beam. Measurement sensors and the engine power system cable are connected to the control system via sliding contacts. A direct current electric motor is created in the conveyor drive. It allows to change the blade drive rotation number of a wide range. The design used in the work may also be used for other purposes, for example, for generation of energy from fluid flow. In this case, like before, all formulas can be used as calculation in relative interaction.
\end{abstract}

Keywords: water transport, conveyor, energy generation.

\section{Introduction}

Various types of devices and mechanisms are used for mixing and moving liquids in large magazines, canals or ponds. The main principle of their operation includes the rotary axis with different shapes of radial wings (impellers). The position of the wings against the rotation axis does not change. The flow of fluid is directed axially (if there is a restrictive cylinder) or axially - radially. For example, some devices are given in [1-4].

In the calculations of these inventions and many other similar structures, the theory of continuous environmental mechanics, combined with analytical approximations or computer technology, is applicable. Examples of such calculations are described in many books and publications [5-10]. The effects of impeller eccentricity and the Reynolds number on the mixing performance were studied for Rushton turbine stirred tank systems operated in the laminar regime $(R e<10)$ [5]. Analysis on energy conversion of a screw centrifugal pump in impeller is studied in [6]. An integrated overview of incompressible flow turbomachines and transport options as pumps, including the theory and experimental studies, are described in the work [7]. Example of vibration analysis of a mixed flow pump impeller blade is given in [8]. Many works are devoted to study of power capability of impellers and the energy conversion mechanism of flow transport mechanisms, and to the methods of theoretical analysis and numerical simulation by computational fluid dynamics theory (CFD), specifically discussing the conditions of internal flow such as velocity, pressure and concentration, for example in $[9 ; 10]$.

From the analysis of scientific articles in classical fluid mechanics, it follows that the main problem in inventing new equipment for fluid transfer is related to the need to study the joint movement of solid bodies with a variable flow $[11 ; 12]$. The analysis of the above studies shows that it would be desirable to develop simplified approximation methods for calculation of tasks by classic mechanics in an integral form (in parallel with the rough calculation of computational fluid dynamics). This work is specifically dedicated to solving the problems described above devoted to the description of the analytical method for fluid and one-sided flat-wing interaction calculation, when additionally the wing starts vibrate. In this method will be used: equations from classical mechanics [13], previous works of authors $[14 ; 15]$ and analysis of flow one part named "added mass" motion [16-18]. 
A new water flow translation motion transporter (conveyor) with tree blades shown in Fig. 1 is investigated. In this device the actuator blades move in the plane axis $\mathrm{O} 1$ and $\mathrm{O} 2$ parallel to cycloidal motion, but the fluid flows away perpendicular to these rotation axis (Fig. 1,2.). For this purpose the blades two component movement is synthesized by kinematics of the gear box: - the first rotation takes place around the central axis $\mathrm{O} 2$ of each blade, while the other rotation of the rotor 1 is around these centres around the central axis $\mathrm{O} 1$ of the transporter. The movement is designed in such a way that the central axis rotates twice as fast as the blades around its axles. This kinematics is shown and explained in Fig. 2.

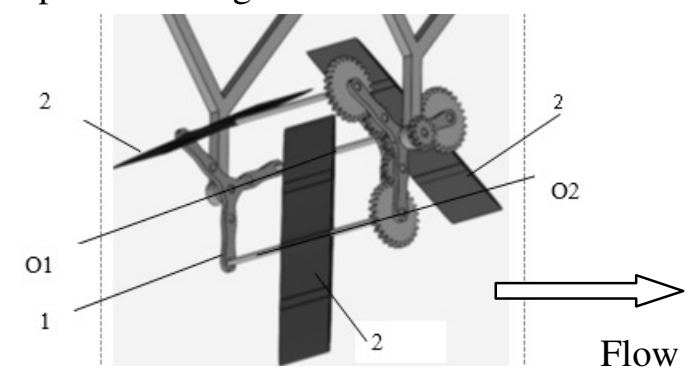

Fig. 1. Model of three blades conveyor:

1 - central rotor with three levers;

2 - blades; $\mathrm{O} 1, \mathrm{O} 2$ - rotation axes

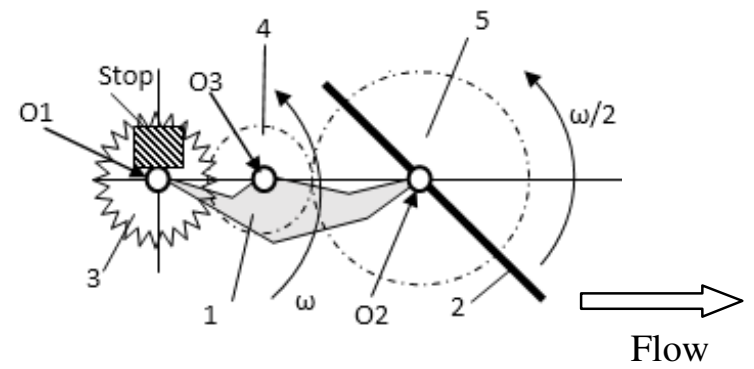

Fig. 2. Kinematics of one blade conveyor: $1-$ central rotor as one lever; 2 - blade; $O 1, O 2$ - rotation axes;

3 - fixed gear (wheel); 4 - moving parasite gear

(wheel); 5 - moving gear (wheel) at which blade is fixed 2

The number of teeth per wheel (gear) 5 is twice the number of teeth per wheel (gear) 3 . Therefore, the wing 2 turns twice slower $(\omega / 2)$ than the central lever $1(\omega)$

\section{Kinematics and dynamics analysis}

One blade motion kinematic is shown in Fig. 3. If the blade in the starting position is perpendicular to $\mathrm{x}$-axis, maximum of interaction forces exist and the flow moves parallel to that axis. After half of turn the blade is parallel to $\mathrm{x}$-axis. It means that interaction forces with fluid are minimal.

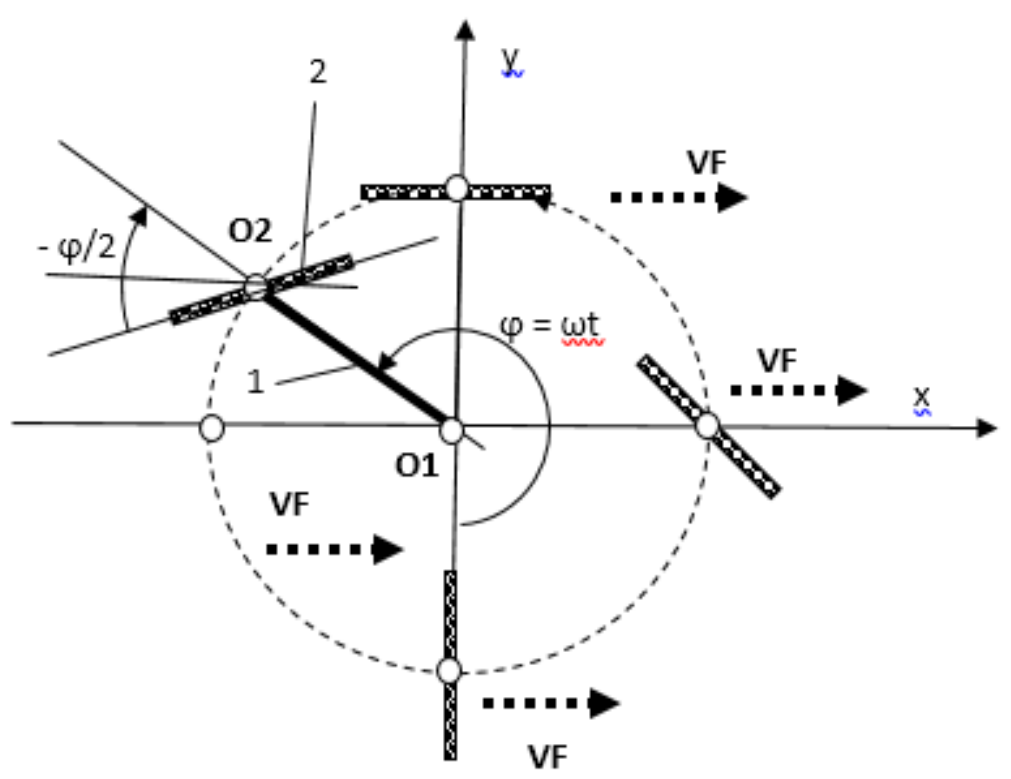

Fig. 3. One blade motion kinematics: 1 - central rotor (lever); 2 - blade; $O 1, O 2$ - rotation axes; $\varphi$ - rotation angle; $x$ - flow direction; $V F$ - middle velocity of flow 


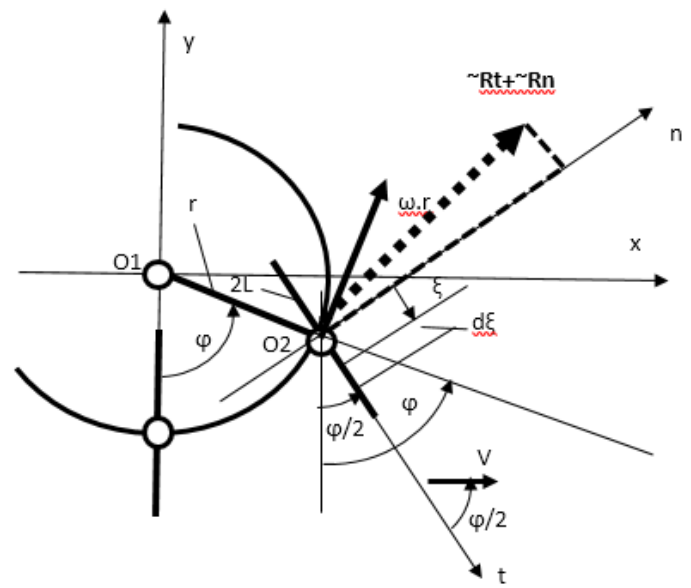

Fig. 4. One blade motion kinematics and dynamics calculation: $r$ - central rotor radius;

$2 L$ - blade length; $O 1, O 2$ - rotation axis;

$\varphi$ - rotation angle; $\mathrm{x}$ - flow direction;

$V=V F-$ velocity of flow positive direction;

$(\sim R t+\sim R n)-$ blade interaction with flow

Dynamic analysis of interaction forces between the blades and flow includes complicated equations of fluid dynamics [11-13]. For simplification, it is possible to use approximated formulas, given in $[14 ; 15]$.

According to [13-15], the interaction force in normal direction (as lifting force) can be calculated by formula (1):

$$
R n=Q 0 \cdot\left\{2 \cdot L \cdot\left[V \cdot \cos \left(\frac{\omega \cdot t}{2}\right)-r \cdot \omega \cdot \cos \left(\frac{\omega \cdot t}{2}\right)\right]^{2}+\frac{L^{3} \cdot \omega^{2}}{6}\right\}
$$

where $Q 0$ - constant parameter including density of fluid, width of plate, drag coefficient in normal direction $[11 ; 12]$;

$\omega$ - angular velocity of central rotor;

$r$ - radius of center $O 2$;

$V$ - flow velocity.

For motion of flow (added mass center) along $x$ axis with projection of force $R n$ (1) the next approximation formula (2) can be used (it is a theorem of classical mechanics of mass center motion [13]):

$$
M \cdot \ddot{X}=-Q 0 \cdot\left\{2 \cdot L \cdot\left[\dot{X} \cdot \cos \left(\frac{\omega \cdot t}{2}\right)-r \cdot \omega \cdot \cos \left(\frac{\omega \cdot t}{2}\right)\right]^{2}+\frac{L^{3} \cdot \omega^{2}}{6}\right\} \cdot\left|\cos \left(\frac{\omega \cdot t}{2}\right)\right|-b \cdot \dot{X}(t),
$$

where $X$-displacement;

$M$ - approximate (added) mass [16-18];

$\ddot{X}$ - acceleration of centre;

$\dot{X}(t)=V(t)$ - velocity of centre approximate (added) mass;

$b$ - linear (added) damping coefficient [16];

$\left|\cos \left(\frac{\omega \cdot t}{2}\right)\right|$ - function, that calculates direction of flow.

Results of modelling equation (2) are shown in Fig. 6-9. 


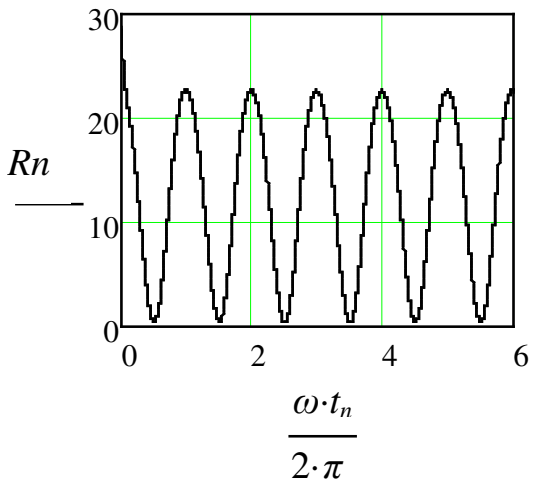

Fig. 6. Interaction force in normal direction (see equation (1)) : $\omega$ - constant angular velocity; $\mathrm{t}$ - time (all parameters in system $\mathrm{SI}$ ); $n$ - computer calculation step

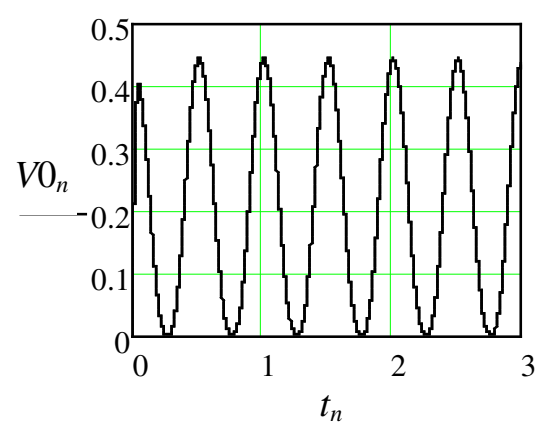

Fig. 8. Velocity of center approximate (added) mass around blade as function of time

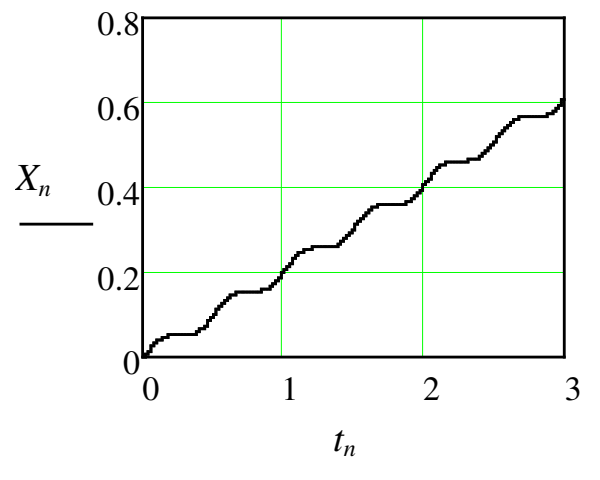

Fig. 7. Displacement of centre approximate (added) mass around blade as function of time (see equation (2)): $X_{n}, x$ - motion direction according to Fig. 3, 4; approximate (added) mass - depends on blade area and real interaction thickness approximation

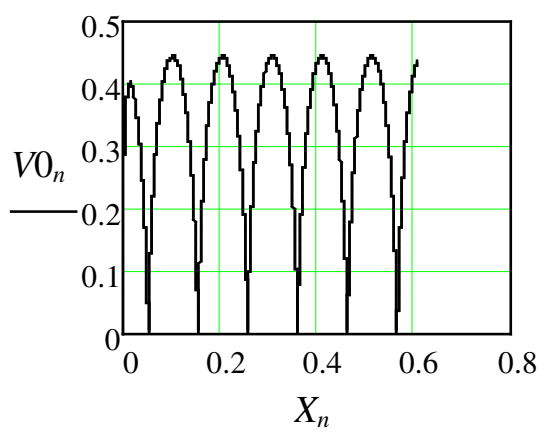

Fig. 9. Motion in phase plane

The possibility to create a multi-element conveyor was considered. In this case, for increase of the efficiency of flow transport many individual small conveyors can be used (Fig. 10.). Here conveyor pairs operate in counter phase, rotating opposite.

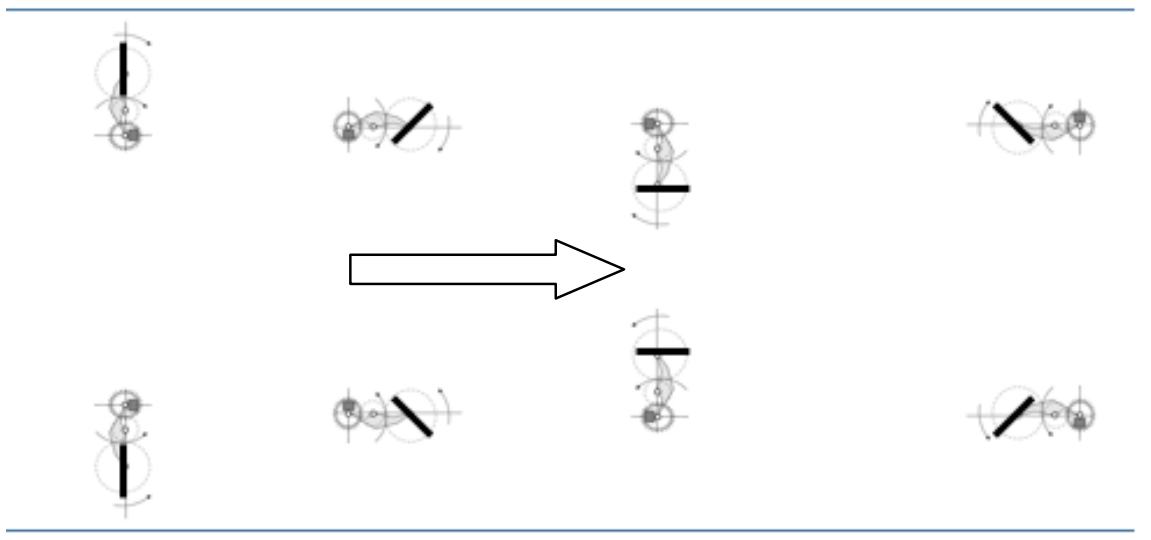

Fig. 10. Multi-element conveyor with eight parts. With given blades rotation directions (upper direct clock direction and lower - indirect clock direction) - flow moves to right side

In that multi-element conveyor motion of the flow center along $x$ axis can be calculated by formula (3) like before by formula (2): 


$$
M \cdot \ddot{X}=\sum_{1}^{k}\left\{-Q 0 \cdot\left\{2 \cdot L \cdot\left[\dot{X} \cdot \cos \left(\frac{\omega \cdot t+\psi_{k}}{2}\right)-r \cdot \omega \cdot \cos \left(\frac{\omega \cdot t+\psi_{k}}{2}\right)\right]^{2}+\frac{L^{3} \cdot \omega^{2}}{6}\right\} \cdot\left|\cos \left(\frac{\omega \cdot t+\psi_{k}}{2}\right)\right|\right\}-b \cdot \dot{X}(t),(3)
$$

where $X$-displacement;

$M$ - full approximate (added) mass around all blades;

$k=(1,2, \ldots-8, \ldots)-$ number of blades elements;

$\psi_{k}$ - phase angle of each blade, for example:

$$
\psi_{1}=\frac{\pi}{4} ; \psi_{2}=\frac{\pi}{2} \psi_{3}=\frac{3 \cdot \pi}{4} ; \psi_{4}=\pi ; \ldots \quad \psi_{8}=2 \cdot \pi . .
$$

For transporter experimental investigations special system inside the water tank was made. The system includes a rotating beam with a possibility to stick the device at the end of this beam. Measurement sensors and the engine power system cable are connected to the control system via the sliding contacts. A direct current electric motor is created in the conveyor drive. It allows to change the blade drive rotation number of a wide range. Some information about that tank is given below in Fig. 11-13.

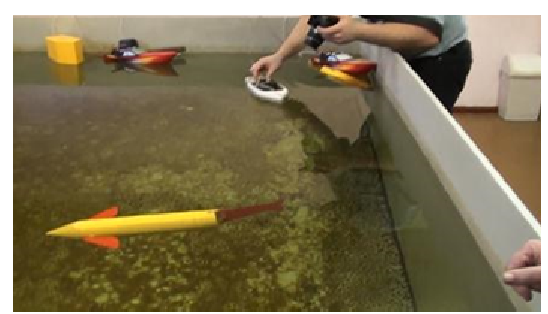

Fig. 11. Water tank with floating devices

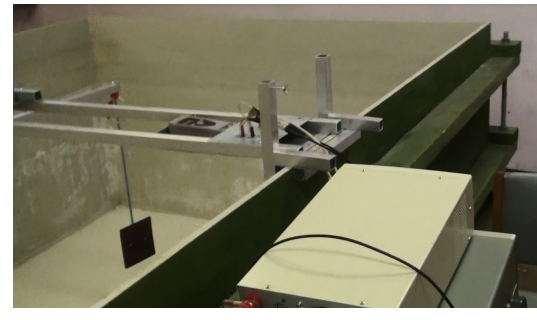

Fig. 12. Unmoving traverse cross tank

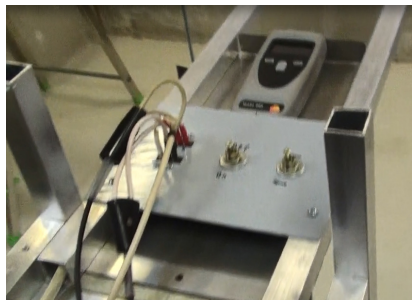

Fig. 13. Parts of power and control system

The design investigated in this work can also be used for other purposes, for example, for generation of energy from fluid flow [15; 19]. In this case, like before, all the same formulas can be used (as calculation in relative interactions with fluid).

\section{Results and discussion}

It is shown how to create a cycloidal motion of conveyor blades so that it is environmentally friendly. For this purpose, the blade movement is synthesised, which does not break the flood and its particles. Looking at the analysis of the given conveyor motion, an approximate analytical method for describing the interaction of a flat plate with moving fluid (for example, water) has been improved. The method allows analytically and then by computer studies to investigate the effect of wing fluctuations on the fluid in the low Reinold's number region, including transitions in the opposite (negative) velocity range. The design used in the work can be applied for other purposes, for example, for generation of energy from fluid flow. In future for accuracy of the results of the given approximate method more experimental investigations will be made and validation given.

\section{Conclusions}

The main conclusions of the report are:

- nature-friendly conveyor model is offered;

- mathematical descriptions of one blade model movement are considered;

- additional mathematical descriptions of multi-element model movement are offered;

- mathematical relationships for productivity calculations are obtained;

- validation water tank for mathematical formulas is made and described.

\section{References}

[1] Asymmetric Blade Impeller. The colorful fluid mixing gallery. 1998. [online] [19.03.2018] Available at: http://www.bakker.org/cfm/bt6.htm. 
[2] Biogas Technology. Efficient pumping and mixing technology for biogas plants. Sundbyberg. Xylem Water Solutions AB, 2012. 16 p. https://swedepump.by/files/biogas.pdf.

[3] Catfish Pond Water Mixer May Decrease Aerator Use. Auburn, Alabama. Auburn University. 1991. http://www.ag.auburn.edu/comm/news/1991/catfish.php.

[4] Howerton R. D., Boyd C.E., Watten B.J. Design and Performance of a Horizontal, Axial-Flow Water Circulator. Journal of Applied Aquaculture. 1994, Vol.3, iss.1-2, pp.163-184.

[5] Cabaret F., Fradette L., Tanguy P. A. Effect of shaft eccentricity on the laminar mixing performance of a radial impeller. Canadian Journal of Chemical Engineering, Dec, 2008, Vol.86(6), $971 \mathrm{p}$.

[6] Quan H., Li R., Su Q., Han W., Wang P. Analysis on Energy Conversion of Screw Centrifugal Pump in Impeller Domain Based on Profile Lines. Advances in Mechanical Engineering, 01 January 2013. Vol.5.

[7] Round G.F. Incompressible Flow Turbomachines. SUBTITLE. Design, Selection, Applications and Theory. Elsevier Science \& Technology. PRINT PUB DATE 2004-07-09 EBOOK PUB DATE. EBOOK ISBN 9780080478456. 364 p.

[8] Divya Z., Apurba R., Kaushik K. Vibration Analysis of Mixed Flow Pump Impeller Blade Designed Using Mean Stream Line Method. Applied Mechanics and Materials, Sep 2016, Vol.852, pp. 476-482.

[9] Punit S., Franz N. Internal hydraulic analysis of impeller rounding in centrifugal pumps as turbines. Experimental Thermal and Fluid Science, 2011, Vol.35(1), pp. 121-134.

[10] Deendarlianto T., Höhne P., Apanasevich D., Christophe L., Matthias V., Matthias B. Application of a new drag coefficient model at CFD-simulations on free surface flows relevant for the nuclear reactor safety analysis. Annals of Nuclear Energy. Volume 39, Issue 1, January 2012, pp. 70-82

[11]Blevins R. D. Applied Fluid Dynamics Handbook. Malabar, Fla.: Krieger, 2003. 570 p. ISBN 978-1-57524-182-1.

[12] Munson B.R., Rothmayer A.P., Okiishi T.H., Huebsch W.W. Fundamentals of Fluid Mechanics. 7th edition. Hoboken, NJ : Wiley, 2012. 792 p. ISBN 978-1-118-11613-5.

[13] Goldstein H., Poole C., Safko J. Classical Mechanics. 3rd edition. Harlow: Pearson, 2014. 638 p. ISBN 978-1-292-02655-8.

[14] Viba J., Eiduks M., Irbe M. Double Pendulum Vibration Motion in Fluid Flow. 14th International Scientific Conference "Engineering for Rural Development", May 20-22, 2015, Jelgava, Latvia: Proceedings, Vol.14. Jelgava: Latvia University of Agriculture, 2015, pp. 434-439.

[15] Viba J., Beresnevich V., Noskovs S., Irbe M. Investigations of Rotating Blade for Energy Extraction from Fluid Flow. Vibroengineering PROCEDIA. 2016, Vol.8, pp.312-315.

[16] Benaouicha M., Astolfi J.A. Analysis of added mass in cavitating flow. Journal of Fluids and Structures, May 2012, Vol.31, pp. 30-48.

[17] Anami K., Ishii N., Knisely C.W. Added mass and wave radiation damping for flow-induced rotational vibrations of skinplates of hydraulic gates. Journal of Fluids and Structures, November 2012, Vol.35, pp. 213-228.

[18] La Mantia M., Dabnichki P. Added mass effect on flapping foil. Engineering Analysis with Boundary Elements. Volume 36, Issue 4, April 2012, pp. 579-590.

[19] Vība J., Noskovs S., Beresņevičs V. Method for Control of Operation Conditions of Wind Turbine and Device for its Realization Vēja turbīnas darba režīma vadības paṇēmiens un ierīce tā realizēšanai. Patent LV 15038 B (Republic of Latvia). Int. Cl. F03D7/06. Application: 27.02.2015 (P-15-21). Date of publication: +20.12.2015. 